\title{
INCREASING THE DEPTH OF THE NITRIDED LAYER IN THE SURFACE OF AUSTENITIC ALLOYS USING FRICTION TREATMENT
}

\author{
V. A. Shabashov*, L. G. Korshunov, A. V. Litvinov, N. V. Kataeva, A. E. Zamatovsky \\ M.N. Miheev Institute of Metal Physics, Ural Branch, Russian Academy of Sciences, 18 S. Kovalevskoy St., \\ Ekaterinburg, Russian Federation \\ *Corresponding author. E-mail: shabashov@imp.uran.ru; address for correspondence: ul. S. Kovalevskoy 18, \\ 620990, Ekaterinburg, Russian Federation
}

In the $\mathrm{Fe}-12 \mathrm{Cr}-30 \mathrm{Ni}$ and $\mathrm{Fe}-15 \mathrm{Cr}-38 \mathrm{Ni}$ ion-plasma nitrided austenitic alloys the depth of the surface, gradient in concentration and phase composition, has been increased from $5 \mu \mathrm{m}$ to $20 \mu \mathrm{m}$ using dry sliding friction and high-pressure torsion in Bridgman anvils. The treatment is based on deformation-induced chromium nitride dissolution-precipitation cyclic phase transitions. As a result of $1 \mathrm{~h}$ nitriding at $500{ }^{\circ} \mathrm{C}$, a nitrogen solution in the $\mathrm{Fe}-\mathrm{Ni}-\mathrm{Cr}-\mathrm{N}$ matrix and disperse nitrides of chromium $\mathrm{CrN}$ and iron $\mathrm{Fe}_{4} \mathrm{~N}$ are formed in the alloy surface layers. Subsequent dry sliding friction or high-pressure torsion leads to nanostructuring, deformation-induced dissolution of chromium and iron nitrides, as well as to the mechanical alloying of the surface and the inner nonnitrided alloy layers. Besides, in the deformed austenitic matrix secondary chromium nitrides are formed. With additional $2 \mathrm{~h}$ annealing at $600{ }^{\circ} \mathrm{C}$, the additional exit of nitrogen from the austenitic $\mathrm{Fe}-\mathrm{Ni}-\mathrm{Cr}-\mathrm{N}$ solid solution occurs, with the preferential formation of $\mathrm{Cr}_{2} \mathrm{~N}$ nitride and the increase of the volume (depth) of the alloy matrix changed in terms of its structure and composition.

The reverse sequence, that is, preliminary friction and subsequent nitriding of the surface, does not noticeably increase the depth of the nitrided layer. It is explained by the accelerated migration of non-equilibrium grain boundaries in the surface nanostructured by friction, which reduces the time required for complete recrystallization to much less than the time of $1 \mathrm{~h}$ nitriding at $500{ }^{\circ} \mathrm{C}$. The result of fast recrystallization is the slight effect of fast diffusion in the surface nanostructured by friction.

Keywords: nitriding, deformation, $\mathrm{Fe}-\mathrm{Cr}-\mathrm{Ni}$ alloys, chromium nitrides.

DOI: $10.17804 / 2410-9908.2016 .6 .017-027$

\section{References}

1. Shabashov V.A., Borisov S.V., Litvinov A.V., Kataeva N.V., Afanas'ev S.V., Titova S.G. Producing a gradient-composition nanocrystalline structure on nitrided surfaces of invar-type $\mathrm{Fe}-\mathrm{Ni}$ alloys using megaplastic deformation. The Physics of Metals and Metallography, 2014, vol. 115, no. 9, pp. 871-883. DOI: 10.1134/S0031918X14090117.

2. Shabashov V.A., Korshunov L.G., Sagaradze V.V., Kataeva N.V., Zamatovsky A.E., Litvinov A.V., Lyashkov K.A. Mossbauer analysis of deformation dissolution of the products of cellular decomposition in high-nitrogen chromium manganese austenite steel. Philosophical Magazine, 2014, vol. 94, no. 7, pp. 668-682. DOI: 10.1080/14786435.2013.859758.

3. Shabashov V.A., Borisov S.V., Litvinov A.V., Zamatovsky A.E., Lyashkov K.A., Sagaradze V.V., Vildanova N.F. Mechanomaking of nanostructure in nitrided $\mathrm{Fe}-\mathrm{Cr}$ alloys by cyclic "dissolution-precipitation" deformation-induced transformations. High Pressure Research, 2013, vol. 33, no. 4, pp. 795-812. DOI: 10.1080/08957959.2013.844230.

4. Korshunov L.G., Shabashov V.A., Chernenko N.L., Pilyugin V.P. Effect of contact stresses on the phase composition, strength, and tribological properties of nanocrystalline structures formed in steels and alloys under sliding friction. Metal Science and Heat Treatment, 2008, vol. 50, no. 11-12, pp. 583-592. DOI: 10.1007/s11041-009-9103-2. 
5. Shabashov V.A., Borisov S.V., litvinov A.V., Zamatovsky A.E., Vil'danova N.F., Voronin V.I., Shepatkovsky O.P. Nanostructure formation and phase transformations in nitrided stainless steel Kh18N8 during severe cold deformation. The Physics of Metals and Metallography, 2009, vol. 107, no. 6, pp. 601-612. DOI: 10.1134/S0031918X09060106.

6. Teplov V.A., Pilyugin V.P., Kuznetsov R.I., Tupitsa D.I., Shabashov V.A., Gundyrev V.M. $\mathrm{BCC} \rightarrow \mathrm{FCC}$ phase translation induced by deformation under pressure in a $\mathrm{Fe}-\mathrm{Ni}$ alloy. Fizika Metallov i Metallovedenie, 1987, vol. 64, no. 1, pp. 93-100. (In Russian).

7. Rusakov V.S. Mesbauerovskaya spektroskopiya lokalno-neodnorodnykh system [Mössbauer Spectroscopy of Locally Inhomogeneous Systems]. Almaty, OPNI IYaF NYaTs RK Publ., 2000, 431 p. (In Russian).

8. Bozort R.M. Ferromagnetizm. Gl. 5. Splavy zhelezo-nikel [Ferromagnetism, D. van Nostrand Company, 1951]. M., Inostrannaya literature Publ., 1975, p. 123. (In Russian).

9. Men'shikov A.Z., Teplykh A.E. Magnetic state diagram of $\gamma$-FeNiCr alloys. Fizika Metallov i Metallovedenie, 1977, vol. 44, no. 6, pp. 1215-1221. (In Russian).

10. Bansal C., Chandra J. Mössbauer studies in disordered $(\mathrm{NiFe})_{1-\mathrm{x}} \mathrm{Cr}_{\mathrm{x}}$ alloys. Solid State Communications, 1976, vol. 19, iss. 2, pp. 107-109. DOI: 10.1016/0038-1098(76)90445-2.

11. Rochegude P., Foct J. Influence of interstitial nitrogen on the thermal expansion of $\mathrm{Fe}_{64} \mathrm{Ni}_{36} \mathrm{~N}_{\mathrm{Xn}}$ alloys. Scripta Metallurgica et Materialia, 1992, vol. 27, iss. 3, pp. 325-328.DOI: 10.1016/0956-716X(92)90520-O.

12. Ali-Zade I.I., Aliyev S.S., Karayeva Sh.N., Shamilov T.G., Shukyurov T.A. Effect of nitriding on the magnetic structure and properties of invar iron-nickel alloys. In: Trudy mezhdunarodnoy konferentsii "Fizika 2005" ["Physics 2005": International Conference Proceedings, Azerbaijan, Baku, 7-9 June, 2005]. Baku, 2005, pp. 394-395. (In Russian).

13. Shabashov V.A., Sagaradze V.V., Morozov S.V., Volkov G.A. A Mossbauer study of the kinetics of deformation-induced dissolution of intermetallics in $\mathrm{Fe}-\mathrm{Ni}-\mathrm{Ti}$ austenite. Metallofizika, 1990, vol. 12, no. 4, pp. 107-114. (In Russian).

14. Kolobov Yu.R., Valiev R.Z., Grabovetskaya G.P., Zhilyaev A.P., Dudarev E.F., Ivanov K.V., Kashin O.A., Naidenkin E.V. Zernogranichnaya diffuziya i svoistva nanostrukturnykh materialov [Grain Boundary Diffusion and Properties of Nanostructured Materials]. Novosibirsk, Nauka Publ., 2001, 232 p. (In Russian).

15. Smolyakova M.Yu., Vershinin D.S., Kolobov Yu.R., Chernikov S.V., Stogney O.V., Tregubov I.M. The effect of nitriding at low temperatures on tribological and magnetic properties of austenitic stainless steel. Inorganic Materials: Applied Research, 2012, vol. 3, iss. 5, pp. 440-445. DOI: $10.1134 / \mathrm{S} 2075113312050164$. 
Подана в журнал: 14.11 .2016

УДК 669.1'24:539.89:543.429.3

DOI: $10.17804 / 2410-9908.2016 .6 .017-027$

\title{
УВЕЛИЧЕНИЕ ГЛУБИНЫ АЗОТИРОВАННОГО СЛОЯ ПОВЕРХНОСТИ АУСТЕНИТНОГО СПЛАВА С ИСПОЛЬЗОВАНИЕМ ФРИКЦИОННОЙ ОБРАБОТКИ
}

\author{
В. А. Шабашов ${ }^{*}$, Л. Г. Коршунов, А. В. Литвинов, Н. В. Катаева, А. Е. Заматовский \\ Институт физики металлов УрО РАН им. М.Н. Михеева, ул. С. Ковалевской, 18, \\ Екатеринбург, Россия
}

\begin{abstract}
*Ответственный автор. Электронная почта: shabashov@imp.uran.ru; адрес для переписки: 620990, Россия, Екатеринбург, ул. С. Ковалевской, 18. ФГБУН ИФМ им. М.Н. Михеева УрО РАН
\end{abstract}

В азотированных ионно-плазменным методом аустенитных сплавах $\mathrm{Fe}-12 \mathrm{Cr}-30 \mathrm{Ni}$ и $\mathrm{Fe}-15 \mathrm{Cr}-38 \mathrm{Ni}$ с использованием сухого трения скольжения и сдвига под давлением в наковальнях Бриджмэна осуществлено увеличение глубины (от 5 до 20 мкм) градиентной по концентрации и фазовому составу поверхности. В основу обработки положены циклические фазовые деформационно-индуцированные переходы растворение-выделение нитридов хрома. В результате азотирования при $500{ }^{\circ} \mathrm{C}, 1$ ч в поверхностном слое сплавов формируется раствор азота в матрице $\mathrm{Fe}-\mathrm{Cr}-\mathrm{Ni}-\mathrm{N}$ и дисперсные нитриды хрома $\mathrm{CrN}$ и железа $\mathrm{Fe}_{4} \mathrm{~N}$. Последующее сухое трение скольжения или сдвиг под давлением приводят к наноструктурированию, деформационному растворению нитридов железа и хрома, а также механическому сплавлению поверхностных и внутренних, неподверженных азотированию, слоев сплава. Кроме того, в деформированной матрице аустенита формируются вторичные нитриды хрома $\mathrm{Cr}_{2} \mathrm{~N}$. При дополнительном отжиге $600{ }^{\circ} \mathrm{C}, 2$ ч происходит дополнительный выход азота из аустенитного $\mathrm{Fe}-\mathrm{Cr}-\mathrm{Ni}-\mathrm{N}$ твердого раствора с преимущественным формированием нитрида $\mathrm{Cr}_{2} \mathrm{~N}$ и увеличением объема (глубины) измененной по составу и структуре матрицы сплава. Обратная последовательность, а именно, предварительное фрикционное воздействия и последующее азотирование поверхности не дают заметного увеличения глубины азотированного слоя. Это объясняется ускоренной миграцией неравновесных границ зёрен в наноструктурированной трением поверхности, приводящей к завершению рекристаллизации за время, существенно меньшее времени азотирования 1 ч при $500{ }^{\circ} \mathrm{C}$. Следствием быстрой рекристаллизации становится незначительным эффект быстрой диффузии в наноструктурированной трением поверхности.

Ключевые слова: азотирование, деформащия, $\mathrm{Fe}-\mathrm{Cr}-\mathrm{Ni}$ сплавы, нитриды хрома.

\section{1. Введение}

В качестве способа модификации поверхности для улучшения функциональных свойств сталей и сплавов широко применяется ионно-плазменное (ИП) напыление и, в частности, азотирование. При ИП-азотировании в поверхностном слое формируются нитриды, распределение которых неоднородно и ограничено по глубине в несколько микрон. В работах $[1,2]$ было обнаружено увеличение глубины распределения нитридов в матрице азотированных сталей с использованием большой холодной пластической деформации фрикционным воздействием и сдвигом под давлением (СД) в наковальнях Бриджмэна. Объяснение этого было дано на основе механизма циклических фазовых деформационноиндуцированных переходов растворение-выделение нитридов [3]. Деформация СД приводит к растворению нитридов $\mathrm{CrN}$, сформированных в поверхностных слоях матрицы, насыщая структуру дефектами $[1,2]$. Последующий отжиг вызывает диффузию азота в объем образца на глубину, существенно превосходящую исходный азотированный слой. Предложенная де-

Shabashov V.A. et al. / Increasing the depth of the nitrided layer in the surface of austenitic alloys using friction treatment 
формационная обработка позволяет получать на поверхности стали упрочненную дисперсными нитридами градиентную наноструктуру с изменяющимся составом и магнитным состоянием матрицы. Деформация СД в определенных отношениях моделирует условия практически значимых интенсивных деформационных воздействий, таких как фрикционное или ударное воздействия на поверхности металлов и сплавов [1-4]. В работе [4] предлагалось рассматривать деформацию СД как макромодель воздействия сухого трения скольжения.

В настоящей работе поставлена задача увеличения глубины градиентной по фазовому и концентрационному составу структуры с использованием растворения частиц нитридов хрома при фрикционной обработке или СД предварительно азотированной поверхности аустенитных нержавеющих сталей $\mathrm{Fe}-\mathrm{Cr}-\mathrm{Ni}$, аналогично $[1-3,5]$.

\section{2. Материалы и методика}

В качестве сплава использовали аустенитные нержавеющие сплавы $\mathrm{Fe}-12 \mathrm{Cr}-30 \mathrm{Ni}$ (мас. \%, 30,4 Ni, 11,8 Cr, ост. Fe) и $\mathrm{Fe}-15 \mathrm{Cr}-38 \mathrm{Ni}$ (мас. \%, 37,6 Ni, 15,2 Cr, ост. Fe). Содержание никеля выбрано так, что в отсутствие хрома сплав будет иметь состав, близкий к составу классического инвара $\mathrm{Fe}-35 \mathrm{Ni}$. Образцами служили прокатанные ( $\psi \sim 60 \%)$ фольги прямоугольной формы толщиной 60...100 мкм, которые подвергались одностороннему ионноплазменному азотированию $[1-3,5]$. Азотирование проводилось в азотно-водородной смеси в диапазоне давлений $0,4 \ldots 1,3$ ГПа. Парциальное давление водорода $8 \times 10^{-2}$ Па. Ток разряда составлял 40-50 А при напряжении на образце 600 В. Температура образца $500{ }^{\circ} \mathrm{C}$, время азотирования - 1 ч. Толщина фольг после азотирования с обеих сторон увеличивалась в пределах десятых долей микрона.

Деформацию азотированной поверхности проводили путем трения скольжения по схеме «цилиндрический индентор (кубический нитрида бора диаметром 7 мм и длиной 5 мм) пластина» при возвратно-поступательном движении индентора рис. 1. Трение осуществляли на воздухе без смазки, при комнатной температуре, при средней скорости скольжения 0,03 м/с и нормальной нагрузке 29,4 Н. Поперечное смещение образца при одном проходе индентора составляло 0,12 мм. Общее количество проходов индентора составляло 100-120. Образцы исследуемых сплавов размером $15 \times 20$ мм были зафиксированы на титановой подложке толщиной 2 мм с использованием механического зажима и клея «Монолит». Коэффициент трения индентора относительно фольги составлял $0,6-0,8$.

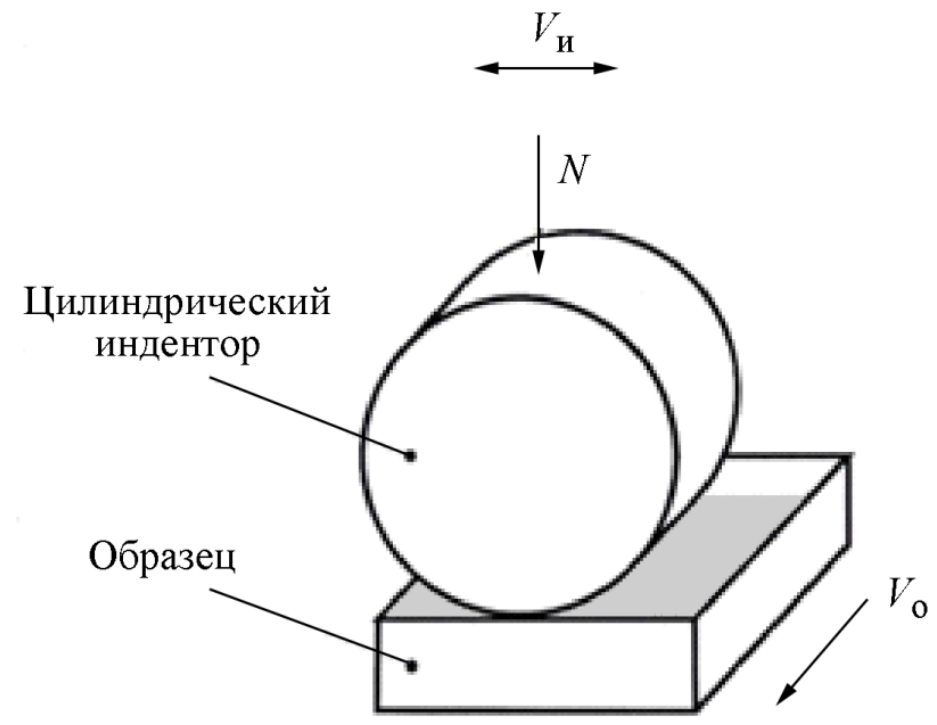

Рис. 1. Схема воздействия - трение скольжения без смазки

Shabashov V.A. et al. / Increasing the depth of the nitrided layer in the surface of austenitic alloys using friction treatment 
Деформацию СД осуществляли по методике, описанной в [6]. Степень истинной деформации при СД оценивали по формуле:

$$
\varepsilon=\varepsilon_{\text {сж }}+\ln \left(1+\frac{\varphi^{2} r^{2}}{d^{2}}\right)^{1 / 2}
$$

где $\varepsilon_{\text {сж }}$ - деформация сжатием; $\varphi=n \times 2 \pi-$ угол поворота наковален при сдвиге $(n-$ число оборотов); $r$ - расстояние от центра образца до исследуемого участка; $d$ - толщина образца

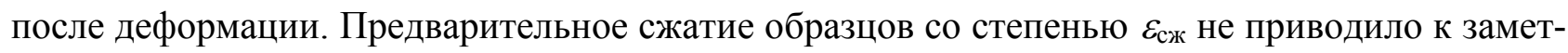
ному изменению мёссбауэровских спектров. Оценку степени деформации $\varepsilon$ по формуле рассматривали как среднюю - с учетом площади образца с определенным радиусом $r$.

Структурное исследование образцов проводили методом мёссбауэровской спектроскопии на прохождение $\gamma$-квантов с энергией 14,4 кЭв от источника ${ }^{57} \mathrm{Co}(\mathrm{Rh})$. Для анализа мёссбауэровских спектров применяли пакет специальных программ [7], улучшающих разрешение спектров. Реставрировали плотность распределения внутренних эффективных полей $\rho(H)$. Для оценки состава матрицы использовали данные по влиянию хрома на магнитную фазовую диаграмму и эффективное магнитное поле аустенита $\mathrm{Fe}-\mathrm{Ni}-\mathrm{Cr}$ сплавов [8-10], а для оценки влияния азота данные работ [11-12]. Выбор толщины фольги - 60...100 мкм, связанный с требованиями фрикционного воздействия и измерения механических свойств, не является оптимальным для трансмиссионной мёссбауэровской спектроскопии вследствие значительного поглощения мёссбауэровского излучения. В связи с этим, в дополнение использовали образцы сплава толщиной 25...30 мкм, оптимальной для мёссбауэровских измерений. Мёссбауэровская спектроскопия дает интегральную по всему объему информацию обо всех неэквивалентных положениях атомов железа в структуре на поверхности и внутри образца, что позволяет проводить количественный фазовый анализ в железосодержащих областях слоистых композитов.

Кроме мёссбауэровской спектроскопии на тех же образцах проводили исследование с использованием рентгеноструктурного анализа (РСА) и трансмиссионной электронной микроскопии (ТЭМ). Рентгеновское исследование осуществлено с помощью дифрактометра ДРОН-4 в $\mathrm{Cu}(\mathrm{K} \alpha)$-излучении. ТЭМ анализ проводился на электронном микроскопе JEM-200CX.

\section{3. Результаты и обсуждение}

Спектр парамагнитного аустенита сплава $\mathrm{Fe}-12 \mathrm{Cr}-30 \mathrm{Ni}$ представляет собой уширенную одиночную линию - синглет (рис. 2 ). Результатом ИП азотирования фольги, прокатанной со степенью $\psi=97$ \% до 30 мкм, также, как и сплава, становится появление в спектре дополнительного секстета с уширенными линиями, полем $\langle H>\sim 250$ кЭ и относительной интегральной интенсивностью до 0,75 (рис. 2 б). ИП азотирование сплава $\mathrm{Fe}-15 \mathrm{Cr}-38 \mathrm{Ni}$ также приводит к формированию в спектре секстета ферромагнитного аустенита (рис. 3 a). Результаты рентгеноструктурного анализа свидетельствуют об образовании в азотированной фольге нитридов $\mathrm{CrN}$ (рис. $4 a$, б). В соответствии с данными по влиянию хрома на магнитные характеристики $\mathrm{Fe}-\mathrm{Cr}-\mathrm{Ni}$ аустенита [8-10], образование ферромагнитного секстета в спектрах объясняется снижением содержания хрома в матрице сплава. Таким образом, секстету в азотированных фольгах соответствует Fe-Ni аустенит, в котором, по результатам PCA, присутствуют нитриды $\mathrm{CrN}$, а синглету - незадействованная при ИП азотировании часть матрицы. Дополнительный отжиг при $600{ }^{\circ} \mathrm{C}$ практически не изменяет интенсивность секстетов, несколько снижая его среднее поле.

Вид спектра фольги толщиной 80 мкм (прокатка с $\psi \sim 60 \%$ ), подвергнутой одностороннему азотированию, в отличие от азотированной фольги в 30 мкм не дает возможности однозначно интерпретировать параметры ферромагнитного секстета вследствие его малой интенсивности (рис. 5 a). Дополнительный отжиг фольги при $600{ }^{\circ} \mathrm{C}, 2$ ч практически не меняет вида спектра.

Shabashov V.A. et al. / Increasing the depth of the nitrided layer in the surface of austenitic alloys using friction treatment 

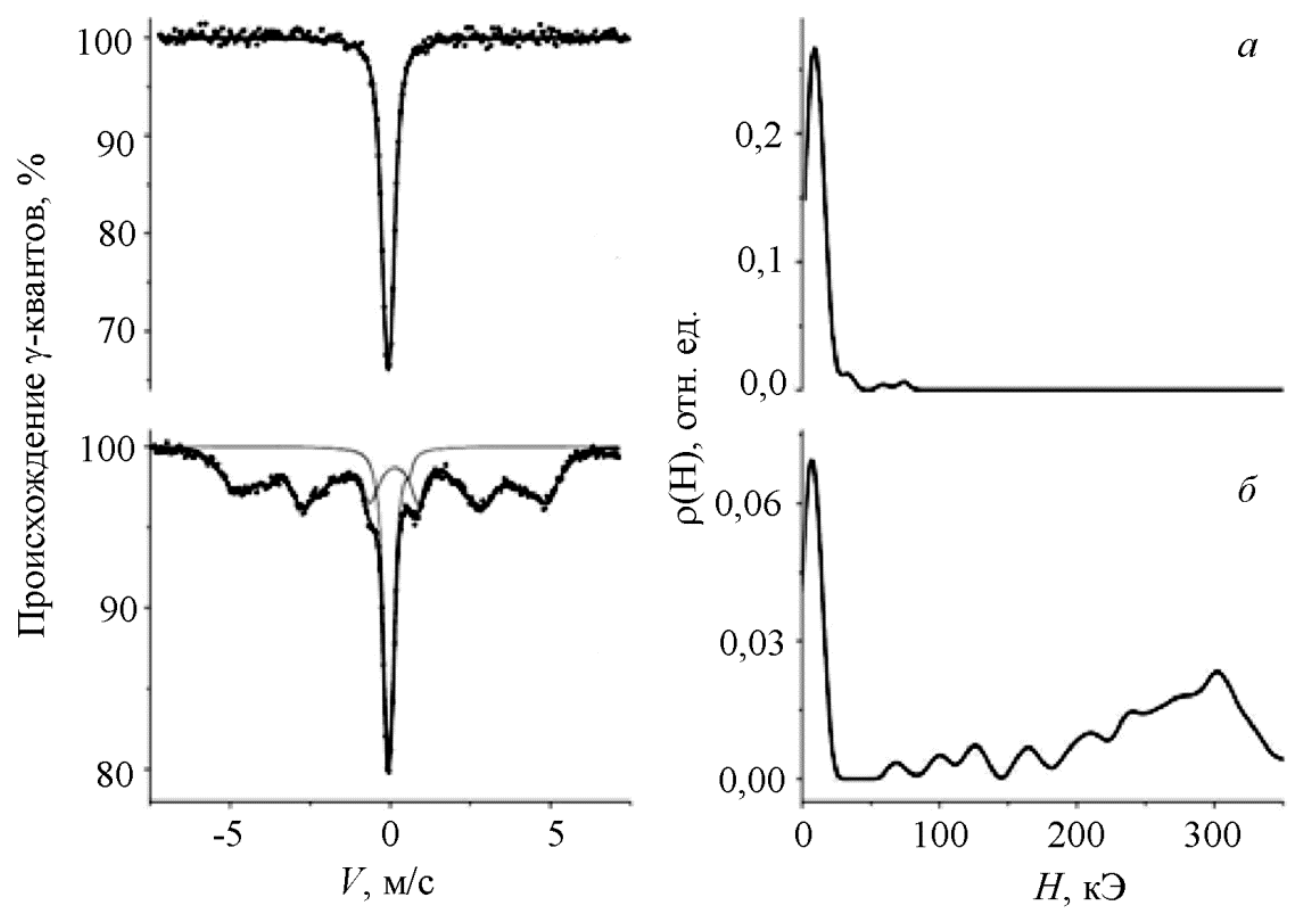

Рис. 2. Мёссбауэровские спектры и распределения $\rho(H)$ фольги сплава $\mathrm{Fe}-12 \mathrm{Cr}-30 \mathrm{Ni}$ толщиной 30 мкм. Обработка последовательно: $a$ - прокатка, $\psi=97 \%$; $\sigma-a+$ одностороннее ИП азотирование

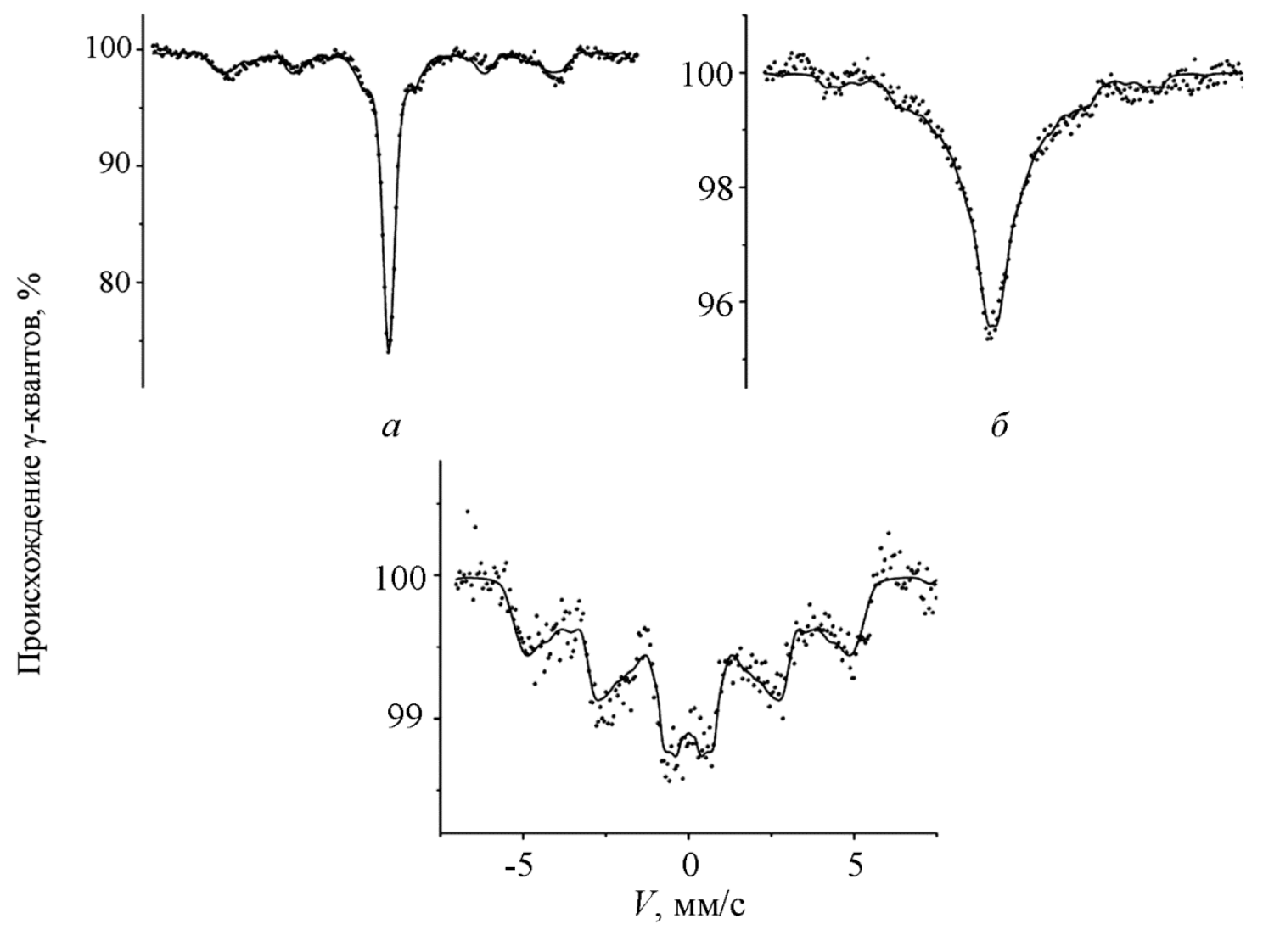

B

Рис. 3. Мёссбауэровские спектры сплава $\mathrm{Fe}-15 \mathrm{Cr}-38 \mathrm{Ni}$. Обработка последовательно: $a$ - прокатка + азотирование; 6 - СД (6 оборотов при 8 ГПа); в - отжиг при $520{ }^{\circ} \mathrm{C}, 2$ ч

Shabashov V.A. et al. / Increasing the depth of the nitrided layer in the surface of austenitic alloys using friction treatment 


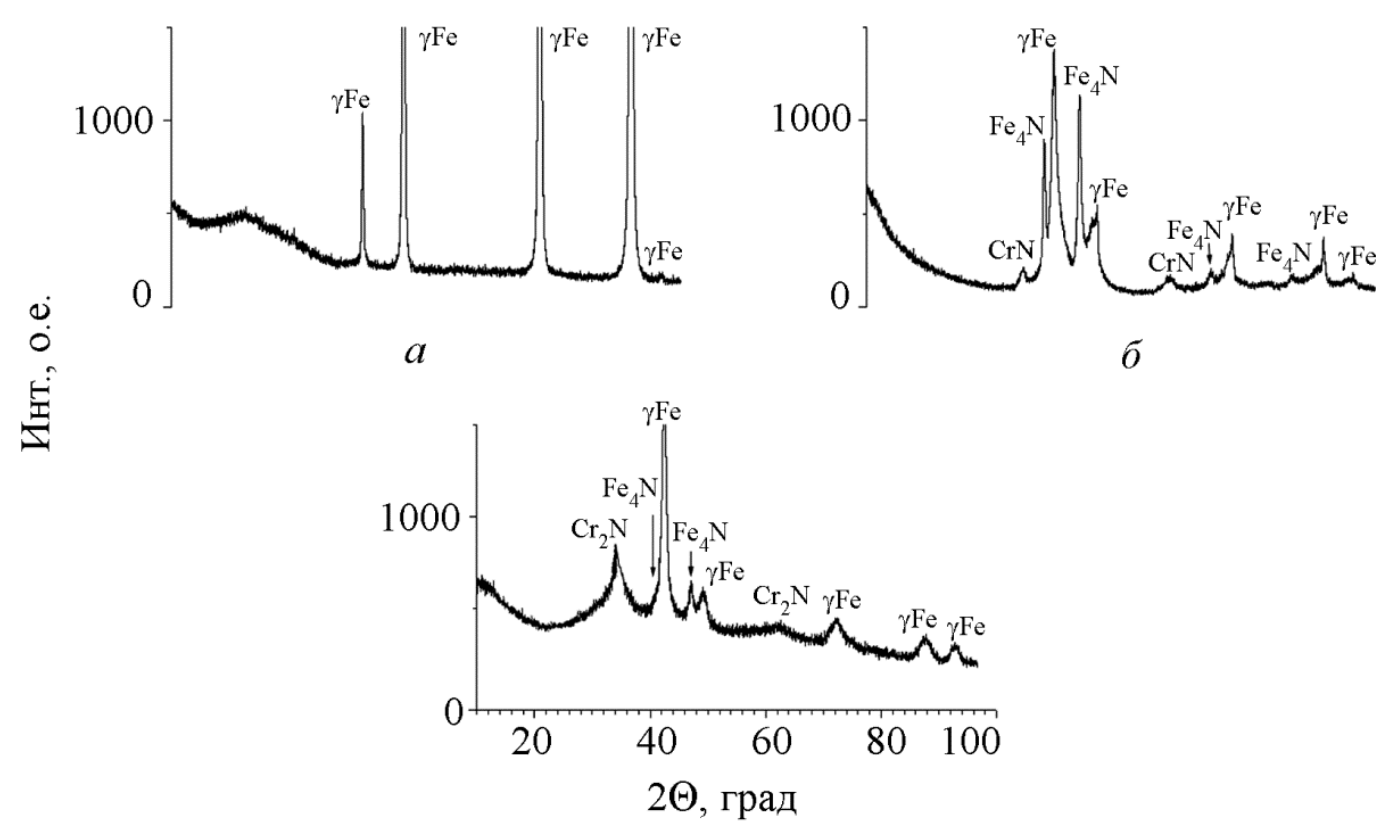

B

Рис. 4. Рентгеновские дифрактограммы сплава $\mathrm{Fe}-15 \mathrm{Cr}-38 \mathrm{Ni}$ после следующих обработок: $a-$ прокатка; $\sigma-a+$ двухстороннее ИП-азотирование; $b-a+\sigma+\mathrm{CД,} 6$ оборотов при 8 ГПа

Результатом фрикционной обработки азотированной поверхности фольги толщиной 80 мкм становится появление в спектре ферромагнитного расщепления, в котором по виду распределения $\rho(H)$ можно выделить пики интенсивности в области полей $\gamma$-(ГЦК), а также $\alpha-(О Ц К)$ фаз (рис. 5 б). В качестве основной причины появления ферромагнитной составляющей следует рассматривать вовлечение дополнительных объемов матрицы в формирование нитридов хрома. В работах $[1-3,5]$ было показано, что трение скольжения и сдвиг под давлением могут приводить к деформационному растворению нитридов $\mathrm{CrN}$ и $\mathrm{Fe}_{4} \mathrm{~N}$ в матрице и способствовать диффузии азота из поверхностных слоев внутрь образца.

Рост количества ферромагнитного аустенита после фрикционного воздействия происходит также вследствие растворения первичных нитридов $\mathrm{Fe}_{4} \mathrm{~N}$ и формирования дополнительных вторичных нитридов с более активным, чем железо, хромом - $\mathrm{CrN}$ и $\mathrm{Cr}_{2} \mathrm{~N}$. Механическое сплавление азотированных приповерхностных и внутренних не азотированных слоев сплава приводит к увеличению общего объема азотированной матрицы и, как следствие, уменьшению интенсивности центральной линии, отвечающей исходному составу сплава (рис. $3 a, \sigma)$.

Наибольшее количество ферромагнитного аустенита (до 0,2 о.е.) в сплаве $\mathrm{Fe}-$ $12 \mathrm{Cr}-30 \mathrm{Ni}$ формируется в подвергнутой трению скольжения фольге после дополнительного отжига при $600{ }^{\circ} \mathrm{C}, 2$ ч (рис. 5 в). Вид спектра, $\rho(H)$ и величина $<H>(\sim 210$ кЭ) ферромагнитной части спектра фольги сплава $\mathrm{Fe}-12 \mathrm{Cr}-30 \mathrm{Ni}$ в этом случае соответствуют бинарному $\mathrm{Fe}-\mathrm{Ni}$ сплаву с содержанием никеля 34,5 ат. \% [13]. Очевидно, что появление ферромагнитного секстета, близкого спектру бинарного $\mathrm{Fe}-\mathrm{Ni}$ сплава инварного состава является следствием дополнительного выхода из матрицы хрома и азота в результате распада аустенита [8-12].

Отжиг при $600{ }^{\circ} \mathrm{C}, 2$ ч ускоряет выход азота и хрома из матрицы с образованием нитридов хрома и способствует переходу метастабильного мононитрида $\mathrm{CrN}$ в нитрид $\mathrm{Cr}_{2} \mathrm{~N}$, что дополнительно понижает содержание хрома в аустените. Подобный отжиг деформированной сдвигом под давлением азотированного сплава $\mathrm{Fe}-15 \mathrm{Cr}-38 \mathrm{Ni}$ приводил к полному исчезновению парамагнитного аустенита и появлению ферромагнитного секстета, соответствующего

Shabashov V.A. et al. / Increasing the depth of the nitrided layer in the surface of austenitic alloys using friction treatment 
http://dream-journal.or"y

бинарному $\mathrm{Fe}-\mathrm{Ni}$ сплаву с содержанием никеля $39 . .40$ ат. \% (рис. 3 в). Фазовый состав сплава и появление нитридов хрома видно на рентгеновской дифрактограмме до и после обработки СД (рис. 4 б, в). Микроструктура сплава после азотирования и обработки СД с последующим сдвигом представлена на рис. 6.
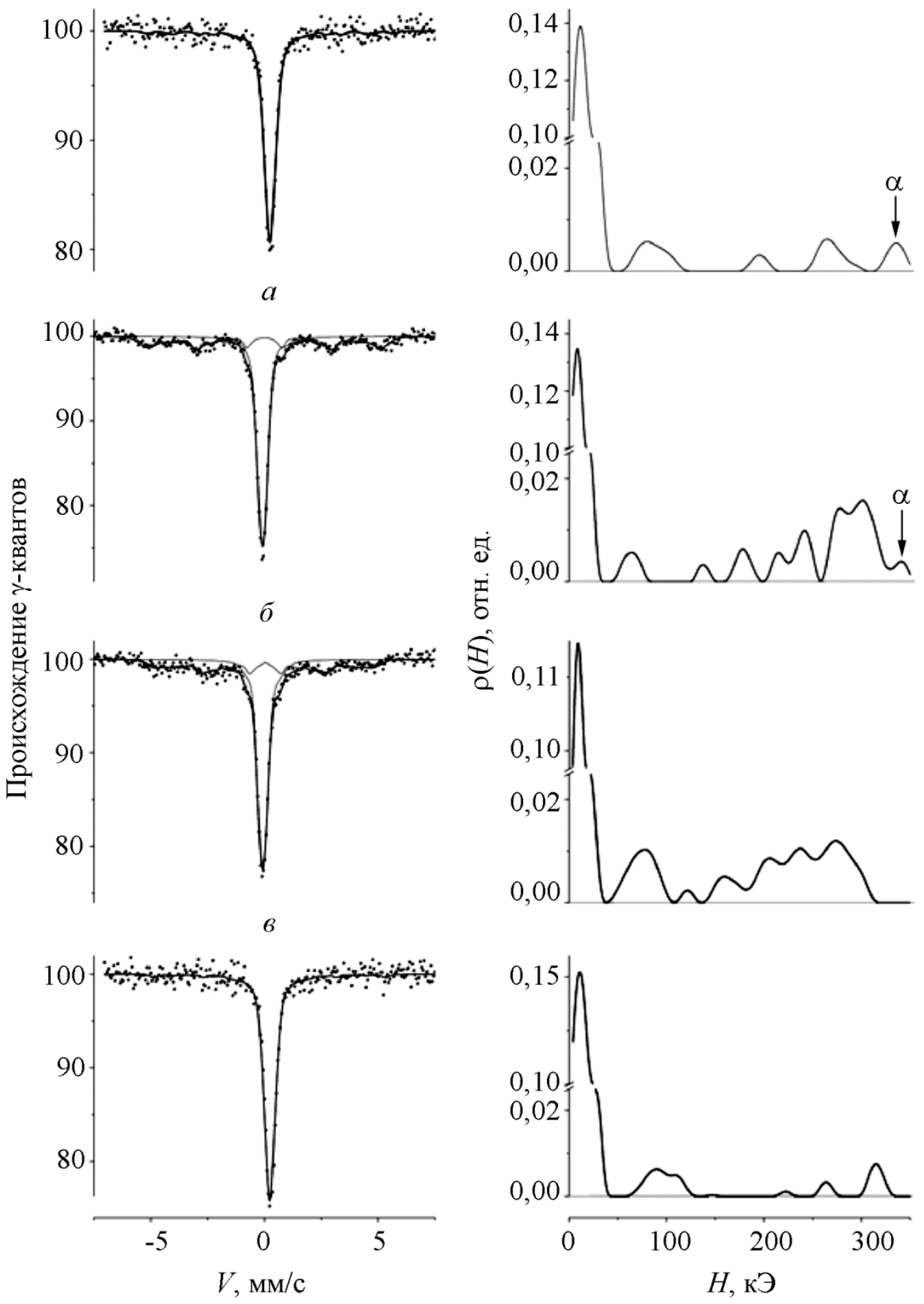

Рис. 5. Мёссбауэровские спектры фольги сплава $\mathrm{Fe}-12 \mathrm{Cr}-30 \mathrm{Ni}$ толщиной 80 мкм. Обработка последовательно: $a$ - прокатка, $\psi=60 \%$ и азотирование; $\sigma$ - сухое трение; $в$ - отжиг $600{ }^{\circ} \mathrm{C}, 2$ ч; 2 - иная последовательность: прокатка; $\psi=60 \%+$ сухое трение + азотирование

Shabashov V.A. et al. / Increasing the depth of the nitrided layer in the surface of austenitic alloys using friction treatment 


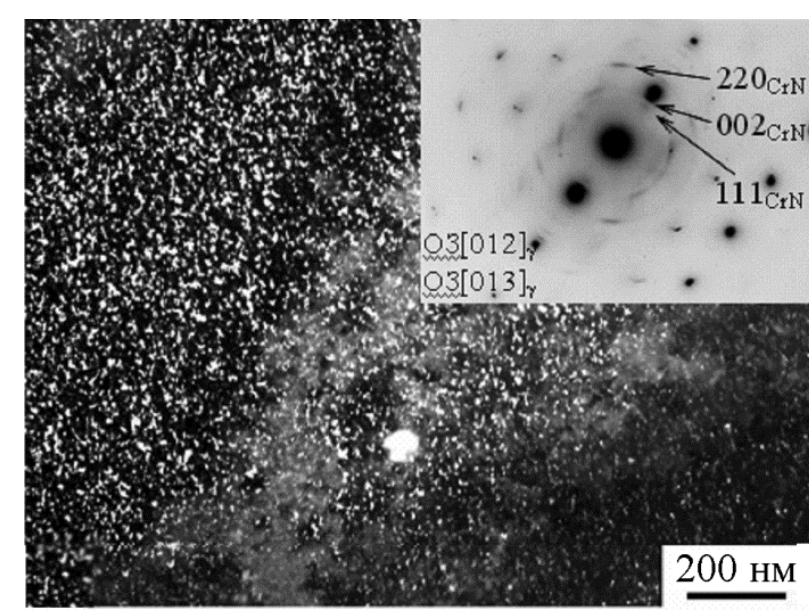

$a$

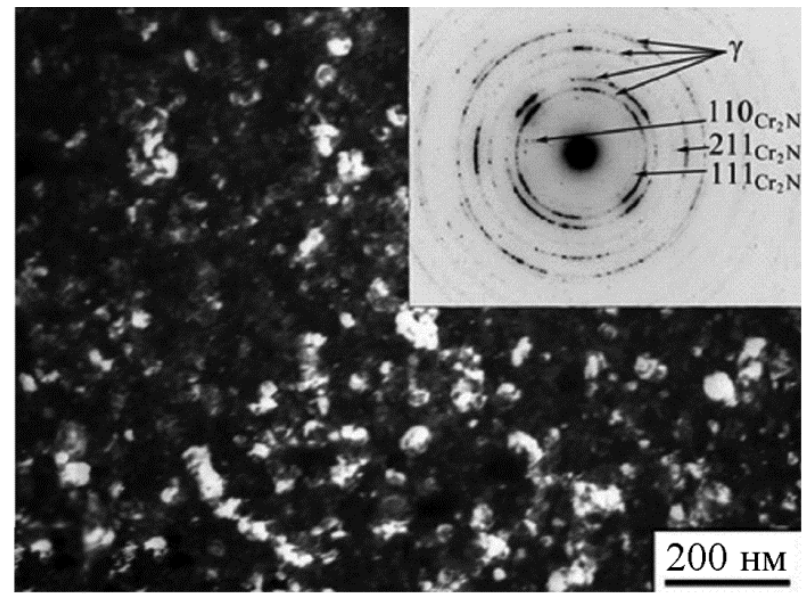

$\sigma$

Рис. 6. Микроструктура сплава $\mathrm{Fe}-15 \mathrm{Cr}-38 \mathrm{Ni}: a$ - прокатка + азотирование; $\sigma-a+\mathrm{CД}+$ отжиг при $520^{\circ} \mathrm{C}$. Приведены темнопольные изображения в рефлексах соответствующих нитридов

Как следует из приведенных выше экспериментальных данных, фрикционная обработка и отжиг азотированной с одной стороны фольги толщиной 80 мкм сплава $\mathrm{Fe}-12 \mathrm{Cr}-30 \mathrm{Ni}$, увеличивают объем ферромагнитной части металлической матрицы до 0,2 о.е. с составом, близким к классическому инвару $\mathrm{Fe}-35 \mathrm{Ni}$. Оценим объем азотированного слоя в зависимости от степени предварительной прокатки фольги. На рис. 2 и и 5 а приведены результаты азотирования сплава $\mathrm{Fe}-12 \mathrm{Cr}-30 \mathrm{Ni}$, прокатанной с различными степенями, а именно, $\psi=97 \%$ (толщина 30 мкм) и $\psi=60 \%$ (толщина 80 мкм). Азотированной фольге толщиной 30 мкм, прокатанной со степенью $\psi=97 \%$ соответствует секстет с интегральной интенсивностью порядка 0,7 о.е. (рис. 2 б), а фольге толщиной 80 мкм, прокатанной со степенью $\psi=60 \%$ - не более $0,05 \ldots 0,1$ (рис. $5 a$ ). Оценивая глубину модифицированного слоя по относительному объему ферромагнитной составляющей, можно сделать заключение, что рост степени прокатки от 60 до 97 \% увеличивает глубину модифицированного слоя от $4 . .10$ до 20 мкм, т.е., более чем в 2 раза. С учетом роста величины самопоглощения резонансного излучения для аустенита, представленного одиночной линией, по отношению к секстету, эта разница может быть ещё более существенной. Таким образом, можно сделать заключение, что деформация поверхности фольги, в том числе с использованием фрикционной обработки, может способствовать активизации диффузии азота в объем сплава и образованию нитридов хрома.

Представляет интерес тот факт, что в мёссбауэровском спектре фольги толщиной 80 мкм процесс ИП-азотирования, проведенный после фрикционной обработки, т.е., в обратной последовательности фрикционной обработки и азотирования, не выявляется заметного (в пределах погрешности измерения) количества ферромагнитной структуры (рис. 5 г). Фрикционное воздействие формирует на глубине 5...10 мкм метастабильную неравновесную структуру, которая содержит значительное число неравновесных границ зерен. В процессе миграции эти границы могут дать аномально быструю зернограничную диффузию при относительно низких температурах $\left(400 \ldots 600{ }^{\circ} \mathrm{C}\right)[14]$. Нагрев образца в процессе ИП-азотирования $\left(500{ }^{\circ} \mathrm{C}\right)$ вызывает рекристаллизацию этой метастабильной наноструктуры благодаря миграции неравновесных границ, которая в свою очередь приводит к ускорению диффузии и завершается после рекристаллизации. Однако если процесс рекристаллизации очень быстрый, по сравнению со временем азотирования (2 ч), эффект быстрой диффузии может быть незначительным. Можно предположить, что в этом случае имеет место быстрая рекристаллизация, приводящая к видимому ограничению объема азотирования.

Shabashov V.A. et al. / Increasing the depth of the nitrided layer in the surface of austenitic alloys using friction treatment 
Как показано в [15], при азотировании аустенита $\mathrm{Fe}-18 \mathrm{Cr}-10 \mathrm{Ni}-\mathrm{Ti}$ диффузия проходит в одном направлении от границ в объем зерна. Нитриды формируются преимущественно вдоль границ зерен. Фрикционное воздействие дробит и растворяет нитриды хрома и железа в поверхностных слоях и приводит к сплавлению этих слоев с более глубокими не азотированными слоями матрицы. Азот из растворенных нитридов железа связывается с более химически активным хромом, образуя дополнительное количество нитридов хрома. Последующий отжиг ускоряет эти процессы и способствует формированию из мононитридов хрома более стабильных нитридов $\mathrm{Cr}_{2} \mathrm{~N}$. Деформационное растворение продуктов ячеистого распада $\left(\mathrm{Cr}_{2} \mathrm{~N}\right)$ в аустенитном сплаве $\mathrm{Fe}-22 \mathrm{Mn}-18 \mathrm{Cr}-0,8 \mathrm{~N}$ с использованием фрикционного воздействия и сдвига под давлением, a также показано увеличение глубины азотированного слоя сплава после фрикционной обработки поверхности было показано в работе [2]. Таким образом, постдеформационный отжиг подвергнутой трением азотированной поверхности фольги приводит к проникновению азота на глубину, существенно превосходящую слой после исходного азотирования. Подтверждением этого является эксперимент по отжигу предварительно деформированной сдвигом под давлением азотированной фольги сплава $\mathrm{Fe}-15 \mathrm{Cr}-38 \mathrm{Ni}$, в котором весь объем образца становится ферромагнитным (рис. 3 в).

Из полученных выше результатов следует, что объем модифицированного слоя матрицы сплавов $\mathrm{Fe}-12 \mathrm{Cr}-30 \mathrm{Ni}$ и $\mathrm{Fe}-15 \mathrm{Cr}-38 \mathrm{Ni}$ с использованием фрикционной обработки или СД с последующим отжигом азотированной поверхности позволяет увеличить объем структуры с измененным концентрационным и фазовым составом. Как следует из структурного мёссбауэровского анализа, в фольге толщиной 80 мкм формируется слой глубиной до 20 мкм состава, близкого к классическому инвару $\mathrm{Fe}-35 \mathrm{Ni}$.

\section{5. Заключение}

Регулирование глубины, фазового и концентрационного состава поверхностных слоев матрицы сплавов $\mathrm{Fe}-12 \mathrm{Cr}-30 \mathrm{Ni}$ и $\mathrm{Fe}-15 \mathrm{Cr}-38 \mathrm{Ni}$ осуществлено с использованием деформационно-индуцированной трением скольжения циклических фазовых переходов «растворениевыделение» нитридов в азотированной поверхности.

ИП азотирование поверхности сплавов $\mathrm{Fe}-12 \mathrm{Cr}-30 \mathrm{Ni}$ и $\mathrm{Fe}-15 \mathrm{Cr}-38 \mathrm{Ni}$ с последующими деформацией трением скольжения или СД и отжигом формируют в объеме наноструктурированной матрицы ферромагнитный аустенит состава $\mathrm{Fe}-(34,5 \ldots 40) \mathrm{Ni}$ в смеси с дисперсными нитридами $\mathrm{CrN}$ и $\mathrm{Cr}_{2} \mathrm{~N}$.

Увеличение глубины азотсодержащего слоя при использовании трения скольжения происходит вследствие насыщения структуры дефектами деформации, механического сплавления азотированной поверхности и не азотированных внутренних слоев, растворения первичных нитридов железа и формирования дополнительного количества нитридов с более активным хромом $-\mathrm{CrN}$ и $\mathrm{Cr}_{2} \mathrm{~N}$.

\section{Благодарность}

Работа выполнена при поддержке РФФИ (проект № 15-08-07947) и гранта УрО РАН (№ 15-9-12-45).

\section{Литература}

1. Producing a gradient-composition nanocrystalline structure on nitrided surfaces of invartype Fe-Ni alloys using megaplastic deformation / V. A. Shabashov, S. V. Borisov, A. V. Litvinov, N. V. Kataeva, S. V. Afanas'ev, S. G. Titova // The Physics of Metals and Metallography. - 2014. Vol. 115, no. 9. - P. 871-883. - DOI: 10.1134/S0031918X14090117.

2. Mossbauer analysis of deformation dissolution of the products of cellular decomposition in high-nitrogen chromium manganese austenite steel / V. A. Shabashov, L. G. Korshunov, 
V. V. Sagaradze, N. V. Kataeva, A. E. Zamatovsky, A. V. Litvinov, K. A. Lyashkov // Philosophical Magazine. - 2014. - Vol. 94, no. 7. - P. 668-682. - DOI: 10.1080/14786435.2013.859758.

3. Mechanomaking of nanostructure in nitrided $\mathrm{Fe}-\mathrm{Cr}$ alloys by cyclic "dissolutionprecipitation" deformation-induced transformations / V. A. Shabashov, S. V. Borisov, A. V. Litvinov, A. E. Zamatovsky, K. A. Lyashkov, V. V. Sagaradze, N. F. Vildanova // High Pressure Research. - 2013. - Vol. 33, no. 4. - P. 795-812. - DOI: 10.1080/08957959.2013.844230.

4. Effect of contact stresses on the phase composition, strength, and tribological properties of nanocrystalline structures formed in steels and alloys under sliding friction. Metal Science and Heat Treatment / L. G. Korshunov, V. A. Shabashov, N. L. Chernenko, V. P. Pilyugin. - 2008. - Vol. 50, no. 11-12. - P. 583-592. - DOI: 10.1007/s11041-009-9103-2.

5. Nanostructure formation and phase transformations in nitrided stainless steel Kh18N8 during severe cold deformation/_V. A. Shabashov, S. V. Borisov, A. V. litvinov, A. E. Zamatovsky, N. F. Vil'danova, V. I. Voronin, O. P. Shepatkovsky // The Physics of Metals and Metallography. 2009. - Vol. 107, no. 6. - P. 601-612. - DOI: 10.1134/S0031918X09060106.

6. Фазовый ОЦК $\rightarrow$ ЦЦК переход, вызываемый деформацией под давлением сплава железо-никель / В. А. Теплов, В. П. Пилюгин, Р. И. Кузнецов, Д. И. Тупица, В. А. Шабашов, В. М. Гундырев // Физика металлов и металловедение. - 1987. - Т. 64. - № 1. - С. 93-100.

7. Русаков В. С. Мёссбауэровская спектроскопия локально-неоднородных систем. Алматы: Изд-во ОПНИ ИЯФ НЯЦ РК, 2000. - 431 с. - ISBN 9965-9111-2-6.

8. Бозорт Р. М. Ферромагнетизм / под ред. Е. Н. Кондорского, Б. Г. Лифшица // М.: Иностранная литература, 1975. - С. 123. - (Гл. 5: Сплавы железо - никель).

9. Men'shikov A. Z., Teplykh A. E. Magnetic state diagram of $\gamma$-FeNiCr alloys // Fizika Metallov i Metallovedenie. - 1977. - Vol. 44, no. 6. - P. 1215-1221.

10. Bansal C., Chandra J. Mössbauer studies in disordered $(\mathrm{NiFe})_{1-\mathrm{x}} \mathrm{Cr}_{\mathrm{x}}$ alloys // Solid State Communications. - 1976. - Vol. 19, iss. 2. - P. 107-109. - DOI: 10.1016/0038-1098(76)90445-2.

11. Rochegude P., Foct J. Influence of interstitial nitrogen on the thermal expansion of $\mathrm{Fe}_{64} \mathrm{Ni}_{36} \mathrm{~N}_{\mathrm{Xn}}$ alloys // Scripta Metallurgica et Materialia. - 1992. - Vol. 27, iss. 3. - P. 325-328. DOI: 10.1016/0956-716X(92)90520-O.

12. Влияние азотирования на магнитную структуру и свойства инварных железо-никелевых сплавов / И. И. Али-Заде, С. С. Алиев, Ш. Н. Караева, Т. Г. Шамилов, Т. А. Шукюров // Международная конференция «Fizika-2005», Азербайджан, Баку, 7-9 июня 2005 г.: труды конференции. - 2005. - С. 394-395.

13. A Mossbauer study of the kinetics of deformation-induced dissolution of intermetallics in Fe-Ni-Ti austenite / V. A. Shabashov, V. V. Sagaradze, S. V. Morozov, G. A. Volkov // Metallofizika. - 1990. - Vol. 12, no. 4. - P. 107-114.

14. Зернограничная диффузия и свойства наноструктурных материалов / Ю. Р. Колобов, Р. З. Валиев, Г. П. Грабовецкая, А. П. Жиляев, Е. Ф. Дударев, К. В. Иванов, М. Б. Иванов, О. А. Кашин, Е. В. Найденкин. - Новосибирск: Наука, 2001. - 232 с.

15. The effect of nitriding at low temperatures on tribological and magnetic properties of austenitic stainless steel / M. Yu. Smolyakova, D. S. Vershinin, Yu. R. Kolobov, S. V. Chernikov, O. V. Stogney, I. M. Tregubov // Inorganic Materials: Applied Research. - 2012. - Vol. 3, iss. 5. P. 440-445. - DOI: 10.1134/S2075113312050164. 